\title{
Despeckling PolSAR Images with a Structure Tensor Filter
}

\author{
Daniel Santana-Cedrés, Luis Gomez, Senior Member, IEEE, Luis Alvarez, \\ Alejandro C. Frery, Senior Member, IEEE
}

\begin{abstract}
In this paper we propose a new despeckling filter for Fully PolSAR (Polarimetric Synthetic Aperture Radar) images defined by $3 \times 3$ complex Wishart distributions. We first generalize the well-known structure tensor to deal with PolSAR data which allows to efficiently measure the dominant direction and contrast of edges. The generalization includes stochastic distances defined in the space of Wishart matrices. Then, we embed the formulation into an anisotropic diffusion-like schema to build a filter able to reduce speckle and preserve edges. We evaluate its performance through an innovative experimental setup that also includes Monte Carlo analysis. We compare the results with a state-ofthe-art polarimetric filter.
\end{abstract}

Index Terms-Synthetic Aperture Radar Polarimetry, Structure Tensor, Despeckling, Monte Carlo.

\section{INTRODUCTION}

$\mathbf{F}$ ULLY POLSAR (Polarimetric Synthetic Aperture Radar) measures the target reflectivity by using four polarization combinations, which provide better scattering measures than monopolarized SAR systems. Due to that, PolSAR is an effective tool to monitoring ground surface and to perform terrain and land use classification [1]

Spaceborne systems such as RADARSAT-2, Gaofen-3, ALOS-PALSAR, Sentinel, or TerraSAR-X provide huge amounts of PolSAR/SAR data of the earth surface through daily continuous observation. Therefore, automatic tools for data analysis are indeed required. However, every SAR image is corrupted with inherent multiplicative noise (speckle) caused by the coherent interference of waves reflected from the many elementary scatterers within the illuminated scene [2]. Speckle is not truly a noise in the signal processing sense as it provides valuable information. However, speckle makes PolSAR images difficult to interpret, and despeckling is often required to improve image segmentation and classification.

Despeckling filters for PolSAR is an active area of research in remote sensing. There are five main classes:

1) Local filters which use local statistical analysis of data to estimate a speckle reduced model, being the Enhanced Lee filter [3] its most prominent example.

2) Methods based on Partial Differential Equations (PDE), which work in the whole image (see [4], [5]).

Manuscript received $* * *$, $* * * *$; revised $* * *$, $* * * *$.

D. Santana-Cedrés, L. Gomez, and L. Alvarez are with the Imaging Technology Center (CTIM), University of Las Palmas de Gran Canaria, Spain, email: dsantana@ctim.es, \{luis.gomez, lalvarez\}@ulpgc.es

A. C. Frery is with the Laboratório de Computação Científica e Análise Numérica (LaCCAN) - Universidade Federal de Alagoas, email: acfrery@laccan.ufal.br
3) Variational methods [6] combine PDE methods with an optimization strategy, and work also globally.

4) Heuristic methods derive from machine learning strategies, and the CNNs (convolutional neural networks) are showing excellent performance [7].

5) Non-local means filters [8] analyze the similarities between image regions (patches), to estimate a set of weights of a standard mean filter (box filter).

See [9] for a recent review on this topic.

Many of these filters were originally designed to deal with Gaussian noise and then adapted to the peculiarities of both the speckle and the PolSAR data. For instance, Deledalle et al. [10] adapted the original non-local means filter to PolSAR data. Torres et al. [11] followed this approach and introduced stochastic distances between PolSAR models. Even the Enhanced Lee filter is adapted to full-channel data from its original design intended for monopolarized data.

Terebes et al. [12] proposed a Perona-Malik type diffusion filter where the diffusion coefficient is computed from the named multiplicative gradient without using stochastic distances. Jiang et al. [13] employed a measure based on the trace of the covariance matrix, while our approach employs all the information it contains. In this work, we use a generalization of the structure tensor to deal with the inherent particularities of PolSAR data. This operator provides a good estimation of the local variability of the PolSAR image in terms of stochastic distances between Wishart distributions. By embedding this structure tensor into an anisotropic diffusion-like schema, we propose a new despeckling filter that preserves the mean and edges while notably reducing image speckle. Additionally, since it relies on linear operations, it preserves the polarimetric signature. To the best of our knowledge the idea of using the structure tensor and stochastic distances to manage the diffusion power of the filter is completely new.

This paper is organized as follows. Section $\Pi$ I recalls the Wishart distribution and presents the structure tensor model. Section IIII details the design of the proposed filter. In Section $[\mathrm{IV}$ we present the results. Section $\mathrm{V}$ concludes this paper.

\section{Wishart Distribution AND THE GENERALIZED STRUCTURE TENSOR}

PolSAR data measures, for each pixel, the scattering as entries of a $2 \times 2$ complex matrix. Such matrices have four distinct complex elements, $S_{\mathrm{Vv}}, S_{\mathrm{VH}}, S_{\mathrm{HV}}$, and $S_{\mathrm{HH}}$, where the component $S_{i j}$ is the backscattered signal for the $i^{\text {th }}$ transmission and $j^{\text {th }}$ is the reception linear polarization, where 
$i$ and $j$ represent the horizontal $(\mathrm{H})$ and the vertical $(\mathrm{V})$ polarizations, respectively. For the case of dealing with a reciprocal medium (the common case in remote sensing [2]), $S_{\mathrm{HV}}=S_{\mathrm{VH}}$. Therefore, the signal backscattered by each resolution cell can be characterized by the complex scattering vector $\boldsymbol{\Omega}=\left[\begin{array}{lll}S_{\mathrm{VV}} & S_{\mathrm{VH}} & S_{\mathrm{HH}}\end{array}\right]^{T}$, where $T$ stands for vector transposition. This random vector can be modeled by a multivariate complex Gaussian distribution [14].

Multilook processing is applied to raw data to reduce speckle and enhance the signal-to-noise ratio. It is performed by averaging $L$ (number of looks) ideally independent acquisitions of the same illuminated scene by the PolSAR sensor, and from that, each observation can be expressed by

$$
\Sigma=\frac{1}{L} \sum_{\ell=1}^{L} \boldsymbol{\Omega}_{\ell} \boldsymbol{\Omega}_{\ell}^{H}
$$

where $H$ denotes the complex conjugate of the transposed vector $\boldsymbol{\Omega}_{\ell}$, with $\ell=1,2, \ldots, L$ scattering vectors. The number of looks $L$ is unique for the whole image and known. If necessary, it may be also estimated from the data. The matrix $\Sigma$ is Hermitian positive definite and it follows a scaled complex Wishart distribution (see [15]).

\section{A. Generalized structure tensor}

We use a generalization of the structure tensor introduced in [16] to PolSAR images. Given a stochastic distance $d_{S}(\cdot, \cdot)$, for $n, m \in\{-h, 0, h\}$ (where $h$ is the interpixel distance) and $(x, y)$ an image point, we define

$$
d_{\sigma}^{n, m}(x, y)=d_{S}\left(I_{\sigma}(x+n, y+m), I_{\sigma}(x-n, y-m)\right),
$$

where $I_{\sigma}$ represents the convolution of the original PolSAR image with a Gaussian kernel $K_{\sigma}$. Then we define the generalization of the structure tensor matrix as

$$
J_{\rho}\left(I_{\sigma}\right) \equiv K_{\rho} *\left(\begin{array}{cc}
\left(d_{\sigma}^{1,0}\right)^{2} & \operatorname{sgn}(s) d_{\sigma}^{1,0} d_{\sigma}^{0,1} \\
\operatorname{sgn}(s) d_{\sigma}^{1,0} d_{\sigma}^{0,1} & \left(d_{\sigma}^{0,1}\right)^{2}
\end{array}\right),
$$

where $s(x, y)=d_{\sigma}^{1,1}(x, y)-d_{\sigma}^{1,-1}(x, y)$ and $\operatorname{sgn}(\cdot)$ is the signum function. The largest eigenvalue of this matrix $\lambda_{\max }\left(J_{\rho}\left(I_{\sigma}\right)\right)(x, y)$ measures the variability of the PolSAR image in a neighborhood of $(x, y)$. The main advantage with respect to other extensions of the structure tensor of vectorvalued images is that the one proposed is adapted to PolSAR data and it uses stochastic distances to account for image variability.

In this work we use the Kullback-Leibler (KL) stochastic distance:

$$
d_{\mathrm{KL}}\left(\Sigma_{1}, \Sigma_{2}\right)=L\left[\frac{\operatorname{tr}\left(\Sigma_{1}^{-1} \Sigma_{2}+\Sigma_{2}^{-1} \Sigma_{1}\right)}{2}-3\right],
$$

where $\operatorname{tr}(\cdot)$ represents the trace operator.

\section{Structure TENSOR FILTER}

We propose a new anisotropic diffusion filter for PolSAR images. Taking the original PolSAR image $I_{0}(x, y)$ as initial guess, the filtered image $I(t, x, y)$ is given by the solution of the partial differential equation

$$
\frac{\partial I}{\partial t}=\operatorname{div}\left(g\left(\sqrt{\lambda_{\max }\left(J_{\rho}\left(I_{\sigma}\right)\right)}\right) \nabla I\right),
$$

where $g(\cdot)$ is an edge-stopping function. Anisotropic diffusion filtering is a classical tool in computer vision originally introduced by Perona and Malik [17]. In the Perona-Malik model edges are preserved because the diffusion is stopped in the points where there is a high variability of the image intensity value. Since the original Perona-Malik model is formulated for scalar images, the differential operator $|\nabla I|$ provides a good estimation of the local variability of the image. In this paper, we deal with PolSAR images where in each point we have a $3 \times 3$ complex matrix described by a Wishart distribution, so in our case we measure the local variability in terms of stochastic distances between Wishart distributions. In that sense the proposed operator $g\left(\sqrt{\lambda_{\max }\left(J_{\rho}\left(I_{\sigma}\right)\right)}\right)$ provides a good estimation of such local variability using stochastic distances. Then, by introducing this estimation in the diffusion coefficient of the model, we observe that the edges of the PolSAR image are preserved. In the experiments presented in this paper we use $g(s)=\left(1+s^{2} / \lambda^{2}\right)^{-1}$ as edge-stopping function, as proposed in [17].

We use a finite difference form of (4) to make the problem discrete:

$$
\frac{I_{i . j}^{n+1}-I_{i . j}^{n}}{\delta t}=\sum_{(k, l) \in \mathcal{N}} \frac{g_{i, j}+g_{i+k, j+l}}{2} \frac{I_{i+k . j+l}^{n}-I_{i . j}^{n}}{h^{2}},
$$

where $I_{i . j}^{n} \approx I(n \cdot \delta t, i \cdot h, j \cdot h)$ and $\mathcal{N}$ is the usual 4 point neighborhood stencil. We observed, experimentally, that $n=$ 100 iterations are enough to attain the asymptotic state of the solution of the differential equation.

\section{EXPERIMENTAL SETUP}

To test the proposed polarimetric filter, we have performed experiments in both simulated and PolSAR data from actual sensors. The results obtained were compared with a stateof-the-art polarimetric non-local means filter [11] (SDNLM: stochastic distance non-local means) which outperforms standard polarimetric filters such as the Refined Lee and the IDAN (intensity-driven adaptive-neighborhood) filters.

The filter ability at reducing speckle and preserving the mean values within homogeneous areas, is assessed by estimating the mean, the standard deviation and the related ENL (equivalent number of looks). As it is known, mean value $(\mu)$ must be preserved after filtering operation, whereas standard deviation of speckle $(\sigma)$ must be notably reduced. As a consequence of that, ENL must increase. A complete assessment of a despeckling filter shall include a measure of edge preservation (through any of the wide metrics available).

We propose a novel method to assess the global performance of the despeckling filter through the estimation of $\mu, \sigma$, and ENL not on selected ROIs (regions of interest), as it is the standard approach, but taking into account that a groundtruth is known for both simulated data and data from an operational sensor. We estimate $\mu, \sigma$, and ENL on complete large 
classes patches. This new filter assessment naturally includes a measure of edge preservation. Indeed, this departures from the classical approach being extremely severe and robust. Moreover, statistics over the whole image are also included, by computing the mean preservation index (MPI):

$$
\mathrm{MPI}=\left|\frac{\mu_{S}-\mu_{F}}{\mu_{S}}\right|,
$$

where $\mu_{S}$ denotes the mean of the original speckled PolSAR image and $\mu_{F}$ for the filtered version.

In the following subsections we describe the results for simulated data and observations from an actual PolSAR sensor.

\section{A. Simulated Data}

Fig. 1(a) shows the $240 \times 240$ pixels phantom with five classes. It contains large patches and fine details. We simulated observations for each class by sampling from the Wishart distributions reported in [11] with $L=3$. This procedure was repeated 2000 times in order to obtain independent images. A Pauli representation of one of them is depicted in Fig. 1(b).

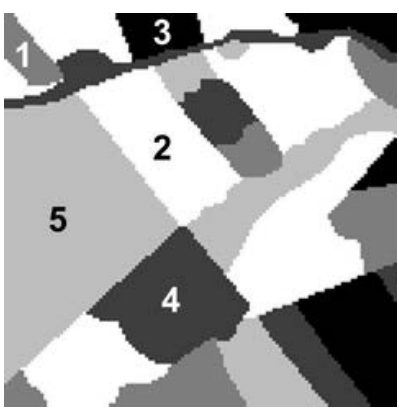

(a) ROIs

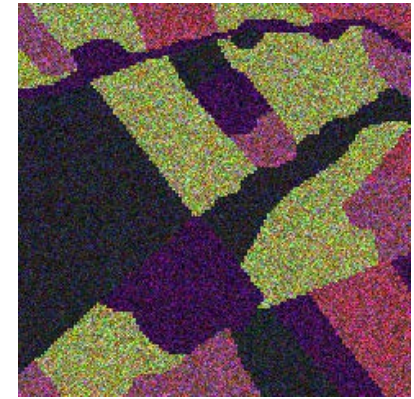

(b) Pauli representation
Fig. 1. Synthetic PolSAR image: (a) the 5 colors of the regions of interest used to compute $\mu$ and $\sigma$, and (b) Pauli codification of a simulated sample.

We processed this dataset with the two polarimetric filters. Fig. 2 shows the results obtained with one of the samples by applying both polarimetric filters (Fig. 2(a) SDNLM and Fig. 2(b) structure tensor filter). Visually, both filters provide excellent results: speckle content has been notably reduced for most classes and edges are well preserved. However, the result from the proposed tensorial filter contains less speckle.

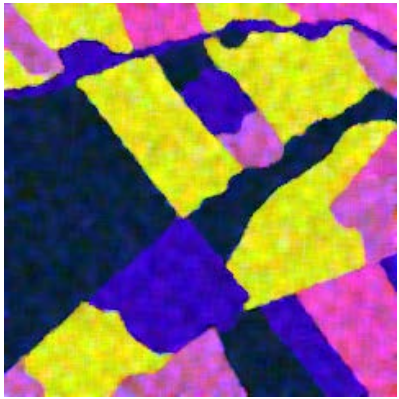

(a) SDNLM

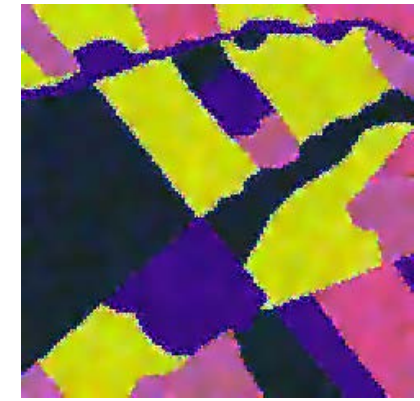

(b) Structure Tensor
Fig. 2. Result of applying:(a) SDNLM and (b) the Structure Tensor filter to the synthetic PolSAR image
Table II presents mean values over the 2000 Monte Carlo replications: $\mu$ and $\sigma$ estimated for the three original bands $\mathrm{HH}, \mathrm{HV}$, and VV. We include the percentage of variation for $\mu$ and $\sigma$ ( $\Delta \mu$ and $\Delta \sigma$, respectively), as well as the mean preservation index (MPI) at the bottom of the table.

As observed, the SDLNM filter presents variations in the mean preservation index, while $\sigma$ is significantly reduced in most of cases, as expected. However, the structure tensor filter shows a more stable performance, with a reduction of the variance in all the bands and classes, including a better preservation of the mean. Note that for the SDNLM filter, there are also cases with a larger than original speckle variance. This is due to the novel assessment used that accounts for the edge preservation. A visual analysis of the results obtained with this non-local means filter shows that edges are not equally preserved for all classes.

Table II shows the equivalent number of looks (ENL). Although SDNLM obtains higher results, they are not uniform, with a worse performance especially in ROIs 4 and 5 . However, the structure tensor filter provides more stable values for all regions (classes).

\section{B. Data from an Operational Sensor}

For the real case we have used the well-known AIRSAR 4 looks intensity PolSAR image from the region of Flevoland in the Netherlands (Fig. 3(a)). Our ground reference consists of 14 ROIs corresponding to different regions, such as crops, urban or water (Fig. 3(b) ; these ROIs are used to compute $\mu, \sigma$, and ENL for the three bands. With this, we are able to evaluate the performance of the despeckling techniques in areas with different polarimetric signatures. These ROIs have been partially based on the ground truth presented in [18], to which we have applied a preprocessing step to remove outliers. Notice that, although just a number is shown near one region, all the ROIs with the same color have been used to compute the statistics.

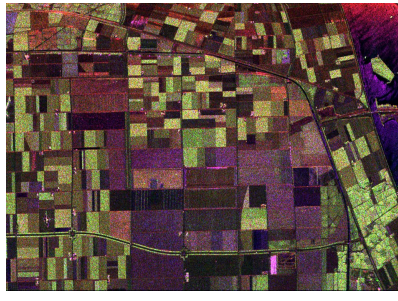

(a) Pauli representation

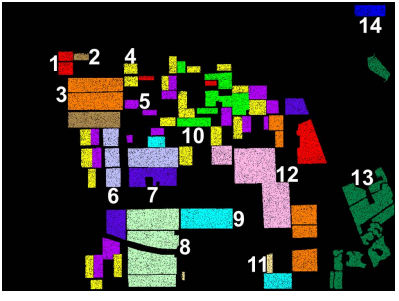

(b) ROIs
Fig. 3. Flevoland PolSAR image: (a) Pauli codification and (b) regions of interest used to compute the statistics.

Fig. 4 shows the results of applying the SDNLM (Fig. 4(a) and structure tensor (Fig. 4(b) filters to the actual PolSAR data. In both cases, we have included in the figure a zoom of an area with different crops. As observed, the SDNLM filter is able to reduce speckle, but the structure tensor provides, in general, a better preservation of the edges and fine details.

Table III follows the same structure as Table II including statistics for the mean and standard deviation, as well as the mean preservation index at the bottom row. Again, the results 
TABLE I

Comparative Results for Synthetic PolSAR Data: Observed data, SDNLM Filter and Structure Tensor Filter

\begin{tabular}{|c|c|c|c|c|c|c|c|c|c|c|c|c|c|c|c|c|c|c|}
\hline \multirow{3}{*}{$\begin{array}{r}\text { Filter } \\
\text { Band } \\
\text { ROI }\end{array}$} & \multicolumn{6}{|c|}{ Observed data $\left(\times 10^{-4}\right)$} & \multicolumn{6}{|c|}{ SDNLM $(\%)$} & \multicolumn{6}{|c|}{ Structure Tensor (\%) } \\
\hline & \multicolumn{2}{|c|}{$\mathrm{HH}$} & \multicolumn{2}{|c|}{ HV } & \multicolumn{2}{|c|}{ VV } & \multicolumn{2}{|c|}{$\mathrm{HH}$} & \multicolumn{2}{|c|}{ HV } & \multicolumn{2}{|c|}{ VV } & \multicolumn{2}{|c|}{$\mathrm{HH}$} & \multicolumn{2}{|c|}{ HV } & \multicolumn{2}{|c|}{ VV } \\
\hline & $\mu$ & $\sigma$ & $\mu$ & $\sigma$ & $\mu$ & $\sigma$ & $\Delta \mu$ & $\Delta \sigma$ & $\Delta \mu$ & $\Delta \sigma$ & $\Delta \mu$ & $\Delta \sigma$ & $\Delta \mu$ & $\Delta \sigma$ & $\Delta \mu$ & $\Delta \sigma$ & $\Delta \mu$ & $\Delta \sigma$ \\
\hline 1 & 36.99 & 21.35 & 5.00 & 2.89 & 62.01 & 35.80 & 2.70 & -77.55 & 0.00 & -60.13 & -1.61 & -79.47 & 0.64 & -73.83 & 3.53 & -62.55 & -0.30 & -74.48 \\
\hline 2 & 56.00 & 32.33 & 18.00 & 10.39 & 55.00 & 31.76 & -1.79 & -83.94 & 0.00 & -84.03 & 0.00 & -82.2 & -0.26 & -69.0 & -0.50 & -68.70 & 0.01 & -69.14 \\
\hline 3 & 45.00 & 25.98 & 4.00 & 2.31 & 70.01 & 40.42 & 0.00 & -82.62 & 0.00 & -22.80 & -1.41 & -84.05 & -0.20 & -74.15 & 1.20 & -69.88 & -0.32 & -74.24 \\
\hline 4 & 11.00 & 6.35 & 1.00 & 0.58 & 24.00 & 13.86 & 9.09 & -37.67 & 0.00 & 20.30 & 4.17 & -72.27 & 0.66 & -52.65 & 3.03 & -50.48 & -0.10 & -52.85 \\
\hline 5 & 9.00 & 5.20 & 2.00 & 1.15 & 13.00 & 7.50 & 11.11 & 20.18 & 0.00 & 110.30 & 7.69 & -19.83 & 0.77 & -70.10 & 0.40 & -69.54 & 1.08 & -69.64 \\
\hline MPI & \multicolumn{2}{|c|}{31.35} & \multicolumn{2}{|c|}{7.69} & \multicolumn{2}{|c|}{39.34} & \multicolumn{2}{|c|}{4.22} & \multicolumn{2}{|c|}{0.00} & \multicolumn{2}{|c|}{1.77} & \multicolumn{2}{|c|}{0.00} & \multicolumn{2}{|c|}{0.00} & \multicolumn{2}{|c|}{0.00} \\
\hline
\end{tabular}

TABLE II

ENL FOR THE SyNTHETIC DATA: SDNLM AND STRUCTURE TENSOR FILTERS

\begin{tabular}{rccccccccc}
\hline Filter & \multicolumn{3}{c}{ Observed data } & \multicolumn{3}{c}{ SDNLM } & \multicolumn{3}{c}{ Structure Tensor } \\
\hline ROIs/Band & HH & HV & VV & HH & HV & VV & HH & HV & VV \\
\hline 1 & 3.00 & 3.00 & 3.00 & 68.93 & 11.70 & 88.26 & 44.40 & 22.92 & 45.81 \\
2 & 3.00 & 3.00 & 3.00 & 84.14 & 69.67 & 95.29 & 31.11 & 30.32 & 31.50 \\
3 & 3.00 & 3.00 & 3.00 & 83.72 & 11.92 & 89.98 & 44.72 & 33.86 & 44.93 \\
4 & 3.00 & 3.00 & 3.00 & 5.58 & 0.73 & 19.06 & 13.56 & 12.99 & 13.47 \\
5 & 3.00 & 3.00 & 3.00 & 6.59 & 3.27 & 12.52 & 34.06 & 32.60 & 33.25 \\
\hline
\end{tabular}

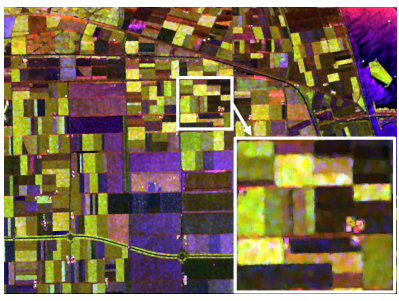

(a) SDNLM

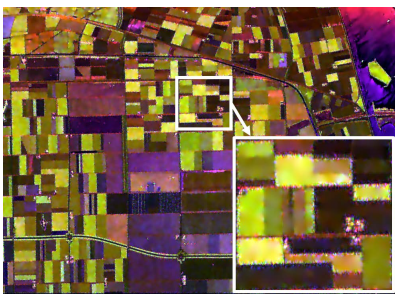

(b) Structure Tensor
Fig. 4. Results of applying (a) the SDNLM filter and (b) the Structure Tensor one to Flevoland PolSAR image.

associated to each filter are presented as the percentage of variation corresponding to the observed data. In most of the ROIs of image processed by the structure tensor filter, the mean preservation is below $0.8 \%$ with slightly more stable performance than in the SDNLM filter. Moreover, $\sigma$ is notably reduced in all cases. In this regard, as in the case of the phantom image, the SDNLM filter presents larger values than the original speckle variance, which reveals a lack of edge preservation. Considering the whole image, as observed at the bottom of the table, the mean preservation index is more stable when the structure tensor filter is applied. Table IV shows the associated ENL over each ROI presented in Fig. 3(b), The results show that the structure tensor filter outperforms the SDNLM in a large number of ROIs.

\section{Implementation Details}

Running our filter, coded in $\mathrm{C}++$ with basic parallel tools, on a $240 \times 240$ pixels image, takes approximately $8 \mathrm{~s}$ on an Intel(R) Core(TM) i7-4870HQ CPU $2.5 \mathrm{GHz}$ (16 GB RAM) computer. The SDNLM filter is coded in Matlab. For the same simulated case, the computational cost is around $70 \mathrm{~s}$ on the same machine (using also basic parallel tools available in Matlab). Both filters are available at http://ctim.ulpgc.es/demo111/

\section{Conclusions}

In this paper, we have introduced a despeckling filter based on a generalization of the structure tensor to the polarimetric SAR case. The formulation of the filter includes a stochastic distances to account for the data variability.

We performed experiments on simulated and data from an actual sensor, and they were compared to a state-of-the-art polarimetric filter: SDNLM. For the first case, 2000 images with $L=3$ were generated and analyzed. In the later, a wellknown PolSAR image was used. The filters were evaluated by computing the mean and standard deviation in multiple regions, as well as the associated equivalent number of looks (ENL). Moreover, we included the mean preservation index (MPI) in the whole image in order to evaluate the mean preservation. We also introduced a new qualitative assessment, which consists on estimating the metrics for large patches of different classes (not user-selected ROIs). This enables the evaluation of the filter performance in areas with different polarimetric signatures in the actual data case, and includes the edge preservation assessment naturally. Considering the obtained results, the proposed filter shows promising outcomes, outperforming the SDNLM in all the metrics evaluated. Future research includes exploring other stochastic distances and differences of entropies, as discussed in [19], [20].

\section{ACKNOWLEDGMENT}

This research has partially been supported by the MINECO project MTM2016-75339-P (Ministerio de Economía y Competitividad, Spain), and by CNPq and Fapeal (Brazil).

\section{REFERENCES}

[1] W. Xie, L. Jiao, and J. Zhao, "PolSAR image classification via DKSVD and NSCT-Domain features extraction," IEEE Geosci. Remote Sens. Lett., vol. 13, no. 2, pp. 227-231, Feb. 2016.

[2] J. S. Lee and E. Pottier, Polarimetric Radar Imaging: From Basics to Applications, 1st ed. Boca Raton, FL, USA: CRC Press, 2009. 
TABLE III

Comparative Results for Flevoland PolSAR Image: Observed Data, SDNLM Filter and Structure Tensor Filter

\begin{tabular}{|c|c|c|c|c|c|c|c|c|c|c|c|c|c|c|c|c|c|c|}
\hline \multirow{3}{*}{$\begin{array}{r}\text { Filter } \\
\text { Band } \\
\text { ROI }\end{array}$} & \multicolumn{6}{|c|}{ Observed data $\left(\times 10^{-4}\right)$} & \multicolumn{6}{|c|}{ SDNLM (\%) } & \multicolumn{6}{|c|}{ Structure Tensor (\%) } \\
\hline & \multicolumn{2}{|c|}{$\mathrm{HH}$} & \multicolumn{2}{|c|}{$\mathrm{HV}$} & \multicolumn{2}{|c|}{ VV } & \multicolumn{2}{|c|}{$\mathrm{HH}$} & \multicolumn{2}{|c|}{$\mathrm{HV}$} & \multicolumn{2}{|c|}{ VV } & \multicolumn{2}{|c|}{$\mathrm{HH}$} & \multicolumn{2}{|c|}{$\mathrm{HV}$} & \multicolumn{2}{|c|}{ VV } \\
\hline & $\mu$ & $\sigma$ & $\mu$ & $\sigma$ & $\mu$ & $\sigma$ & $\Delta \mu$ & $\Delta \sigma$ & $\Delta \mu$ & $\Delta \sigma$ & $\Delta \mu$ & $\Delta \sigma$ & $\Delta \mu$ & $\Delta \sigma$ & $\Delta \mu$ & $\Delta \sigma$ & $\Delta \mu$ & $\Delta \sigma$ \\
\hline 1 & 103.76 & 65.66 & 2.35 & 1.42 & 148.17 & 91.74 & -0.35 & -52.08 & -20.65 & -55.49 & -0.27 & -55.44 & 1.31 & -56.66 & -0.60 & -57.17 & 1.11 & -61.44 \\
\hline 2 & 106.87 & 69.66 & 20.12 & 14.30 & 71.82 & 46.28 & 0.34 & -53.51 & 1.42 & -45.85 & 0.05 & -53.37 & 0.22 & -55.06 & 1.51 & -46.91 & -0.13 & -53.02 \\
\hline 3 & 11.38 & 8.39 & 0.83 & 0.59 & 21.92 & 15.92 & 2.66 & -32.09 & -5.10 & 17.83 & 1.50 & -46.98 & 0.33 & -51.74 & -0.11 & -34.17 & 0.01 & -57.26 \\
\hline 4 & 117.89 & 78.68 & 71.12 & 49.32 & 127.24 & 84.08 & 0.01 & -59.44 & 1.27 & -52.37 & -0.76 & -59.11 & -0.39 & -57.53 & 0.74 & -53.76 & -1.04 & -57.57 \\
\hline 5 & 146.92 & 98.06 & 57.65 & 37.22 & 124.66 & 79.60 & 0.36 & -48.61 & 1.64 & -55.19 & -1.15 & -54.65 & 0.07 & -47.48 & 1.68 & -52.01 & -1.36 & -52.63 \\
\hline 6 & 22.84 & 16.07 & 3.78 & 2.96 & 19.20 & 14.26 & 3.70 & -33.04 & 4.47 & -3.91 & 4.80 & -23.91 & 1.08 & -36.95 & 0.62 & -29.27 & 1.31 & -30.77 \\
\hline 7 & 69.53 & 44.79 & 21.45 & 15.24 & 65.29 & 41.83 & 1.54 & -43.40 & 3.03 & -39.41 & 0.33 & -54.78 & 0.72 & -52.62 & 2.24 & -40.86 & 0.28 & -54.59 \\
\hline 8 & 40.27 & 28.74 & 7.22 & 4.88 & 69.25 & 44.33 & 2.75 & -31.10 & 1.28 & -32.97 & 1.17 & -46.94 & 0.86 & -51.24 & 0.51 & -52.61 & -0.21 & -62.15 \\
\hline 9 & 90.38 & 56.49 & 19.39 & 12.22 & 131.76 & 79.10 & -0.19 & -56.29 & 8.35 & 155.29 & -0.12 & -59.15 & -0.77 & -64.22 & 0.35 & -53.90 & -0.67 & -66.99 \\
\hline 10 & 34.99 & 21.56 & 5.25 & 3.24 & 71.92 & 43.54 & 0.58 & -63.90 & -0.08 & -57.71 & 0.03 & -67.37 & 0.50 & -67.90 & 0.60 & -64.90 & -0.05 & -73.09 \\
\hline 11 & 26.59 & 17.18 & 5.12 & 4.04 & 18.82 & 12.40 & 0.92 & -51.53 & 1.17 & -39.56 & 2.05 & -39.86 & 0.07 & -59.10 & 0.53 & -50.17 & 0.63 & -49.97 \\
\hline 12 & 44.45 & 27.24 & 11.16 & 7.21 & 89.96 & 53.78 & -0.09 & -65.81 & -0.51 & -55.73 & -0.34 & -68.98 & -0.33 & -71.63 & -0.71 & -63.82 & -0.58 & -75.31 \\
\hline 13 & 21.09 & 13.43 & 4.44 & 3.05 & 42.62 & 26.79 & 0.00 & -61.74 & -0.17 & -51.87 & -0.24 & -62.57 & -0.28 & -67.46 & 0.06 & -58.12 & -0.43 & -66.06 \\
\hline 14 & 1262.56 & 1369.68 & 35.16 & 25.16 & 148.33 & 120.10 & 11.70 & -30.69 & -1.51 & -49.98 & 5.65 & -44.27 & 7.26 & -17.22 & 0.00 & -21.29 & 2.57 & -25.60 \\
\hline MPI & & & 17 & & & & & & 0 & & & & & & & & & \\
\hline
\end{tabular}

TABLE IV

ENL For the Actual PolSAR Data: SDNLM And Structure Tensor Filters

\begin{tabular}{|c|c|c|c|c|c|c|c|c|c|}
\hline \multirow{2}{*}{$\begin{array}{l}\text { Filter } \\
\text { Band }\end{array}$} & \multicolumn{3}{|c|}{ Observed data } & \multicolumn{3}{|c|}{ SDNLM } & \multicolumn{3}{|c|}{ Structure Tensor } \\
\hline & $\mathrm{HH}$ & HV & VV & HH & $\mathrm{HV}$ & VV & $\mathrm{HH}$ & $\mathrm{HV}$ & VV \\
\hline 1 & 2.50 & 2.76 & 2.61 & 10.80 & 8.76 & 13.06 & 13.65 & 14.84 & 17.94 \\
\hline 2 & 2.35 & 1.98 & 2.41 & 10.96 & 6.94 & 11.09 & 11.71 & 7.24 & 10.88 \\
\hline 3 & 1.84 & 1.95 & 1.90 & 4.20 & 1.26 & 6.95 & 7.95 & 4.49 & 10.38 \\
\hline 4 & 2.25 & 2.08 & 2.29 & 13.65 & 9.40 & 13.49 & 12.35 & 9.87 & 12.46 \\
\hline 5 & 2.24 & 2.40 & 2.45 & 8.56 & 12.35 & 11.65 & 8.15 & 10.77 & 10.64 \\
\hline 6 & 2.02 & 1.64 & 1.81 & 4.85 & 1.94 & 3.44 & 5.19 & 3.32 & 3.88 \\
\hline 7 & 2.41 & 1.98 & 2.44 & 7.75 & 5.73 & 11.99 & 10.89 & 5.92 & 11.88 \\
\hline 8 & 1.96 & 2.18 & 2.44 & 4.37 & 4.99 & 8.87 & 8.40 & 9.82 & 16.97 \\
\hline 9 & 2.56 & 2.52 & 2.78 & 13.35 & 0.45 & 16.59 & 19.68 & 11.93 & 25.12 \\
\hline 10 & 2.63 & 2.62 & 2.73 & 20.44 & 14.62 & 25.64 & 25.80 & 21.52 & 37.64 \\
\hline 11 & 2.39 & 1.61 & 2.30 & 10.38 & 4.50 & 6.64 & 14.33 & 6.54 & 9.32 \\
\hline 12 & 2.66 & 2.39 & 2.80 & 22.73 & 12.08 & 28.87 & 32.86 & 18.02 & 45.35 \\
\hline 13 & 2.46 & 2.13 & 2.53 & 16.83 & 9.14 & 17.99 & 23.14 & 12.14 & 21.79 \\
\hline 14 & 0.85 & 1.95 & 1.53 & 2.21 & 7.57 & 5.48 & 1.43 & 3.15 & 2.90 \\
\hline
\end{tabular}

[3] J. Lee, T. L. Ainsworth, Y. Wang, and K. Chen, "Polarimetric SAR speckle filtering and the extended sigma filter," IEEE Trans. Geosci. Remote Sens., vol. 53, no. 3, pp. 1150-1160, Mar. 2015.

[4] Y. Yu and S. T. Acton, "Speckle reducing anisotropic diffusion," IEEE Trans. Image Proc., vol. 11, no. 11, pp. 1260-1270, Nov. 2002

[5] L. Gomez, L. Alvarez, L. Mazorra, and A. C. Frery, "Fully PolSAR image classification using machine learning techniques and reactiondiffusion systems," Neurocomputing, vol. 255, pp. 52-60, 2017.

[6] X. Nie, H. Qiao, and B. Zhang, "A variational model for PolSAR data speckle reduction based on the Wishart distribution," IEEE Trans. Image Proc., vol. 24, no. 4, pp. 1209-1222, Apr. 2015.

[7] Z. Zhang, H. Wang, F. Xu, and Y. Jin, "Complex-valued convolutional neural network and its application in polarimetric SAR image classification," IEEE Trans. Geosci. Remote Sens., vol. 55, no. 12, pp. 7177-7188, 2017.

[8] A. Buades, B. Coll, and J. Morel, "A review of image denoising algorithms, with a new one," Multiscale Model. \& Simul., vol. 4, no. 2, pp. $490-530,2005$.

[9] J. Lee, T. L. Ainsworth, and Y. Wang, "A review of polarimetric SAR speckle filtering," in Proc. IGARSS, Jul. 2017, pp. 5303-5306.

[10] C.-A. Deledalle, L. Denis, F. Tupin, A. Reigber, and M. Jäger, "NL-SAR: A unified nonlocal framework for resolution-preserving (Pol)(In)SAR denoising," IEEE Trans. Geosci. Remote Sens., vol. 53, no. 4, pp. 20212038, Apr. 2013.

[11] L. Torres, S. J. Sant'Anna, C. da Costa Freitas, and A. C. Frery, "Speckle reduction in polarimetric SAR imagery with stochastic distances and nonlocal means," Pattern. Recognit., vol. 47, no. 1, pp. 141-157, 2014

[12] R. Terebes, M. Borda, R. Malutan, C. Germain, L. Bombrun, and I. Ilea "PolSAR image denoising using directional diffusion," in 12th IEEE Int. Symp. on Electron. and Telecommun. (ISETC). IEEE, 2016, pp. 339342 .
[13] C. Jiang, X. F. He, L. J. Yang, J. Jiang, D. Y. Wang, and Y. Yuan, "Despeckling PolSAR images based on relative total variation model," in ISPRS - International Archives of the Photogrammetry, Remote Sensing and Spatial Information Sciences, vol. XLII-3. Copernicus GmbH, Apr. 2018, pp. 641-644.

[14] S. N. Anfinsen, A. P. Doulgeris, and T. Eltoft, "Estimation of the equivalent number of looks in polarimetric synthetic aperture radar imagery," IEEE Trans. Geosci. Remote Sens., vol. 47, pp. 3795-3809, 2009.

[15] L. Gomez, L. Alvarez, L. Mazorra, and A. C. Frery, "Classification of complex Wishart matrices with a diffusion-reaction system guided by stochastic distances," Philos. Trans. Roy. Soc. A, Math., Phys. Eng. Sci., vol. 373 , no. 2056, pp. 1-14, Dec. 2015 .

[16] L. Gomez, L. Alvarez, and A. C. Frery, "Local edginess measures in PolSAR imagery by using stochastic distances," in Proc. IGARSS, Jul. 2018, pp. 5800-5803.

[17] P. Perona and J. Malik, "Scale-space and edge detection using anisotropic diffusion," IEEE Trans. Patt. Anal. Machine Intell., vol. 12, no. 7, pp. 629-639, 1990.

[18] S. Uhlmann and S. Kiranyaz, "Integrating color features in polarimetric SAR image classification," IEEE Trans. Geosci. Remote Sens., vol. 52, no. 4, pp. 2197-2216, 2014.

[19] A. C. Frery, A. D. C. Nascimento, and R. J. Cintra, "Analytic expressions for stochastic distances between relaxed complex Wishart distributions," IEEE Trans. Geosci. Remote Sens., vol. 52, no. 2, pp. 1213-1226, 2014.

[20] A. C. Frery, R. J. Cintra, and A. D. C. Nascimento, "Entropy-based statistical analysis of PolSAR data," IEEE Trans. Geosci. Remote Sens., vol. 51, no. 6, pp. 3733-3743, June 2013. 